\title{
Translationally invariant cumulants in energy cascade models of turbulence
}

\author{
Hans C. Eggers ${ }^{1,2}$, Thomas Dziekan ${ }^{3}$ and Martin Greiner ${ }^{2,3,4}$ \\ ${ }^{1}$ Department of Physics, University of Stellenbosch, 7600 Stellenbosch, South \\ Africa \\ ${ }^{2}$ Max-Planck-Institut für Physik komplexer Systeme, Nöthnitzer Str. 38, D-01187 \\ Dresden, Germany \\ ${ }^{3}$ Institut für Theoretische Physik, Technische Universität, D-01062 Dresden, \\ Germany \\ ${ }^{4}$ Department of Physics, Duke University, Durham, NC 27ro8, USA
}

\begin{abstract}
In the context of random multiplicative energy cascade processes, we derive analytical expressions for translationally invariant one- and two-point cumulants in logarithmic field amplitudes. Such cumulants make it possible to distinguish between hitherto equally successful cascade generator models and hence supplement lowest-order multifractal scaling exponents and multiplier distributions.
\end{abstract}

Although the underlying hydrodynamic equations are deterministic, the statistical description of fully developed turbulence has by now a long tradition [1]. Random multiplicative energy cascade models form a particularly simple and robust class of such statistical models. While different theoretical models can reproduce experimentally observed multifractal scaling exponents rather easily [2], observed multiplier distributions [3,4] eliminate many candidate cascade generators. Nevertheless, a number of competing generators remain, equally successful in reproducing both scaling exponents and multipliers. To make further progress in ferreting out the best cascade generator within this approximation, new observables are clearly called for.

While most experiments have concentrated on measuring statistics in the energy dissipation density $\varepsilon$, we recently found a complete and analytical solution working in $\ln \varepsilon$ rather than $\varepsilon$ itself [5]. In this letter, we show that cumulants in $\ln \varepsilon$ are analytically calculable even when restoring translational invariance to the solutions to emulate the spatial homogeneity of experimental turbulence statistics. Cumulants turn out to be powerful tools which for third and fourth 
order differ not only in magnitude but even in sign for the relevant cascade generators and can hence be expected to distinguish between different models which are otherwise indistinguishable in terms of observed lowest-order multifractal scaling exponents and multiplier distributions.

In the simplest versions of random multiplicative energy cascade models, energy flux densities $\varepsilon$ are generated as follows: in successive steps $j=1, \ldots, J$, the integral scale $L$ is divided into equal intervals of length $l_{j}=l_{j-1} / 2=L / 2^{j}$ and dyadic addresses $\kappa=\left(k_{1} \cdots k_{j}\right)$ with $k_{i}=0$ or 1 . At each step $j$, the energy flux $\varepsilon_{k_{1} \cdots k_{j}}$ generates fluxes in the two subintervals via

$$
\varepsilon_{k_{1} \cdots k_{j} k_{j+1}}=q_{k_{1} \cdots k_{j} k_{j+1}} \varepsilon_{k_{1} \cdots k_{j}}
$$

where the random variables $q_{L}=q_{k_{1} \cdots k_{j} 0}$ and $q_{R}=q_{k_{1} \cdots k_{j} 1}$ for the left and right subintervals are drawn from a given cascade-generating probability density $p\left(q_{L}, q_{R}\right)$, independently of other branches and generations of the dyadic tree. When after $J$ cascade steps the smallest scale $\eta=l_{J}=L / 2^{J}$ is reached, the local amplitudes of the flux density field $\varepsilon_{t(\kappa)}$ are interpreted as the energy dissipation amplitudes at positions $1 \leq t(\kappa)=\left(1+\sum_{j=1}^{J} k_{j} 2^{J-j}\right) \leq 2^{J}$ in units of $\eta$, which are to be compared to experimental time series converted to onedimensional spatial series by the frozen flow hypothesis.

We have shown previously that, since the product of multiplicative weights $\varepsilon_{k_{1} k_{2} \cdots k_{J}}=\prod_{j=1}^{J} q_{k_{1} \cdots k_{j}}$ becomes additive on taking the logarithm, $\ln \varepsilon_{k_{1} k_{2} \cdots k_{J}}=$ $\sum_{j=1}^{J} \ln q_{k_{1} \ldots k_{j}}$, the multivariate cumulant generating function for $\ln \varepsilon$ has the analytical solution [5],

$$
\begin{aligned}
\ln Z\left(\lambda_{0 \cdots 0}, \ldots, \lambda_{1 \cdots 1}\right) & =\ln \left\langle\exp \left(\sum_{k_{1}, \ldots, k_{J}=0}^{1} \lambda_{k_{1} \cdots k_{J}} \ln \varepsilon_{k_{1} \cdots k_{J}}\right)\right\rangle \\
& =\sum_{j=1}^{J} \sum_{k_{1}, \ldots, k_{j-1}=0}^{1} Q\left(\lambda_{k_{1} \cdots k_{j-1} 0}, \lambda_{k_{1} \cdots k_{j-1} 1}\right)
\end{aligned}
$$

where the branching cumulant generating function $Q$ (with arguments $\lambda_{k_{1} \cdots k_{j}}=$ $\left.\sum_{k_{j+1}, \ldots, k_{J}=0}^{1} \lambda_{k_{1} \cdots k_{J}}\right)$ is the Mellin transform of the cascade generator,

$$
Q\left(\lambda_{L}, \lambda_{R}\right)=\ln \left[\int d q_{L} d q_{R} p\left(q_{L}, q_{R}\right) q_{L}^{\lambda_{L}} q_{R}^{\lambda_{R}}\right]
$$

which can often be found analytically. A host of analytical predictions for statistics in $\ln \varepsilon$ for a given cascade generator follow, starting with multivariate cumulants obtained directly from $\ln Z$ through

$$
C\left(\kappa_{1}, \kappa_{2}, \cdots, \kappa_{n}\right)=\left\langle\ln \varepsilon_{\kappa_{1}} \ln \varepsilon_{\kappa_{2}} \cdots \ln \varepsilon_{\kappa_{n}}\right\rangle_{c}=\left.\frac{\partial^{n} \ln Z}{\partial \lambda_{\kappa_{1}} \partial \lambda_{\kappa_{2}} \cdots \partial \lambda_{\kappa_{n}}}\right|_{\lambda=0} .
$$


Due to the additivity of $\ln Z$ in (2), these cumulants in $\ln \varepsilon$ become simple sums [5] of same-lineage cumulants $c_{n}$ and splitting cumulants $c_{r, s}$ in $\ln q$,

$$
\begin{gathered}
c_{n}=\left\langle(\ln q)^{n}\right\rangle_{c}=\left.\frac{\partial^{n} Q}{\partial \lambda_{L}^{n}}\right|_{\lambda_{L}=\lambda_{R}=0}, \\
c_{r, s}=\left\langle\left(\ln q_{L}\right)^{r}\left(\ln q_{R}\right)^{s}\right\rangle_{c}=\left.\frac{\partial^{r+s} Q}{\partial \lambda_{L}^{r} \partial \lambda_{R}^{s}}\right|_{\lambda_{L}=\lambda_{R}=0},
\end{gathered}
$$

where without loss of generality we have assumed $Q\left(\lambda_{L}, \lambda_{R}\right)$ to be symmetric in its arguments. When all $n$ addresses are the same, the $n$-th order theoretical one-point cumulant is simply

$$
C_{n}(\kappa)=\left\langle\left(\ln \varepsilon_{\kappa}\right)^{n}\right\rangle_{c}=J c_{n}
$$

while the theoretical two-point cumulant of order $(r, s)$ for bins $\kappa_{1}=\left(k_{1} \cdots k_{j} k_{j+1} \cdots k_{J}\right)$ and $\kappa_{2}=\left(k_{1} \cdots k_{j} k_{j+1}^{\prime} \cdots k_{J}^{\prime}\right)$ with $k_{j+1} \neq k_{j+1}^{\prime}$, separated by the ultrametric distance $D=J-j$, is given by [5]

$$
C_{r, s}(D)=\left\langle\left(\ln \varepsilon_{\kappa_{1}}\right)^{r}\left(\ln \varepsilon_{\kappa_{2}}\right)^{s}\right\rangle_{c}=(J-D) c_{r+s}+\left(1-\delta_{D, 0}\right) c_{r, s}
$$

Three- and higher-point cumulants take on a form very similar to the twopoint expression [5].

Before theoretical cumulants can be compared to experimentally observed ones, two complications must be dealt with. The first is that the generating function (2) and its cumulants are not translationally invariant, in conflict with the homogeneous statistics characterising experimental results. The second complication arises because experimental cumulants are derived from measured moments rather than the other way round [6], requiring translational averaging over two-point moments rather than two-point cumulants for theory also. The proper procedure is hence to convert theoretical cumulants (8) to moments, average these to restore translational invariance, and then convert them back to translationally invariant cumulants for experimental comparison.

For one-point statistics, this procedure is trivial, yielding translationally averaged one-point cumulants $\bar{C}_{n}=J c_{n}$. Although the latter cannot distinguish between a spatially ordered cascade process and one whose field amplitudes are spatially randomised, useful information can nevertheless be extracted from them. To show this we introduce three factorised model cascade generators,

$$
p\left(q_{L}, q_{R}\right)=p\left(q_{L}\right) p\left(q_{R}\right)
$$


which have proven successful in reproducing multifractal scaling exponents, namely a simple binomial weight distribution

$$
p_{\text {binomial }}(q)=\frac{\alpha_{2}}{\alpha_{1}+\alpha_{2}} \delta\left(q-\left(1-\alpha_{1}\right)\right)+\frac{\alpha_{1}}{\alpha_{1}+\alpha_{2}} \delta\left(q-\left(1+\alpha_{2}\right)\right)
$$

with parameters $\alpha_{1}=0.3$ and $\alpha_{2}=0.65$, a lognormal distribution

$$
p_{\text {lognormal }}(q)=\frac{1}{\sqrt{2 \pi} \sigma q} \exp \left[-\frac{1}{2 \sigma^{2}}\left(\ln q+\frac{\sigma^{2}}{2}\right)^{2}\right]
$$

with parameter $\sigma=0.42$, and a Beta distribution

$$
p_{\text {Beta }}(q)=\frac{\Gamma\left(\beta_{1}+\beta_{2}\right)}{\Gamma\left(\beta_{1}\right) \Gamma\left(\beta_{2}\right)} 8^{1-\beta_{1}-\beta_{2}} q^{\beta_{1}-1}(8-q)^{\beta_{2}-1}
$$

with parameters $\beta_{1}=4.88=\beta_{2} / 7$ and $q \in[0,8]$. All quoted parameter values were obtained from fitting to the observed multiplier statistics, including scale correlations [4].

As shown in Fig. 1a, all three cascade generators yield almost identical results for the lowest-order multifractal scaling exponents $\tau(n)=\log _{2}\left\langle q^{n}\right\rangle$. Since $\langle q\rangle=$ 1 by construction, $\tau(1)=0$ for all three distributions. For $n=2$ we get $\tau(2)=0.26$ for the first two distributions and 0.23 for the beta distribution, i.e. indistinguishable within the uncertainty of the experimental intermittency exponent $\mu=0.25 \pm 0.05[2]$. Note that the $\tau(n)$ 's for the binomial and the Beta distributions remain indistinguishable even for $n \geq 3$.

While the multiplier distributions and lowest-order scaling exponents of the three cascade generators (10)-(12) are hence demonstrably indistinguishable, Fig. $1 \mathrm{~b}$ shows that, while $c_{1}$ and $c_{2}$ are very similar for the three models, the same-lineage cumulants exhibit clear-cut differences in third order, with respective values $c_{3}=0.05,0.00$ and -0.05 . In fourth order, the same-lineage cumulant $c_{4}$ also exhibits a different sign for the binomial and Beta distribution. For the lognormal distribution, $c_{4}$ is of course again zero.

The relation between the exponents and cumulants is easily demonstrated using the cumulant branching generating function (3):

$$
c_{n}=\left.\frac{\partial^{n} Q(\lambda, 0)}{\partial \lambda^{n}}\right|_{\lambda=0}=\left.\frac{\partial^{n} \ln \left\langle q^{\lambda}\right\rangle}{\partial \lambda^{n}}\right|_{\lambda=0}=\left.\ln 2 \frac{\partial^{n} \tau(\lambda)}{\partial \lambda^{n}}\right|_{\lambda=0},
$$

$\overline{1}$ We note that other popular log-stable or log-Poisson weight distributions are good at reproducing multifractal scaling exponents but fail to account for the proper scale-correlations observed in multiplier distributions [4]. 
i.e. the cumulant $c_{n}$ in $\ln q$ is the $n$-th derivative of the scaling exponent $\tau(\lambda)$ at $\lambda=0$. In principle, therefore, the complete set of same-lineage cumulants $c_{n}$ with $1 \leq n<\infty$ contains the same information as the complete set of multifractal exponents $\tau(n)$ with $0 \leq n<\infty$ : for the former case an inverse Mellin transformation recovers the (factorised) cascade generator, while the latter uses the inverse Laplace transform. Once, however, we truncate the two sets of observables to the lowest orders, $1 \leq n \leq 4$, they sample different aspects of the complete information. This is the reason why the three cascade generators given above can be hard to distinguish by means of lowest-order multifractal scaling exponents while still exhibiting significant differences for the thirdand higher-order same-lineage cumulants.

As mentioned previously, one-point statistics are not sensitive to the nested spatial hierarchy of the cascade. By contrast, eq. (8) shows that two-point cumulants depend on the cascade geometry through the ultrametric distance $D$. To access such spatial information, we now concentrate on two-point cumulants.

For two-point moments, spatial homogeneity can be emulated by creating a theoretical time series consisting of a chain of $m \rightarrow \infty$ independent cascade fields with $L / \eta=2^{J}$ finest-scale bins each [7]. While this scheme is simple, it has previously played a decisive role in explaining scale-correlations for observed multiplier distributions [3,4] and observed Markov properties [8,9]. For two-point statistics with constant bin-bin distance $d<L / \eta$, the appropriate averaging is given by

$$
\bar{\rho}_{r, s}(d)=\lim _{m \rightarrow \infty} \frac{1}{(m-1) 2^{J}} \sum_{t=1}^{(m-1) 2^{J}}\left(\ln \varepsilon_{t}\right)^{r}\left(\ln \varepsilon_{t+d}\right)^{s}
$$

where $t$ and $t+d$, corresponding to $\kappa_{1}$ and $\kappa_{2}$ of (8), are in base-ten notation. Operationally, these bins are moved over the series in bin-sized steps, successively "seeing" parts of adjacent cascade configurations. For $d<L / \eta$, the $t$-average over the infinitely long chain of the independently and identically distributed cascade configurations can hence be replaced by a combination of an ensemble average \langle\rangle and a $t$-average restricted to two adjacent cascade configurations, so that (14) simplifies to $\bar{\rho}_{r, s}(d)=2^{-J} \sum_{t=1}^{2^{J}} \rho_{r, s}(t, t+d)$, where $\rho_{r, s}(t, t+d)=\left\langle\left(\ln \varepsilon_{t}\right)^{r}\left(\ln \varepsilon_{t+d}\right)^{s}\right\rangle$. Since furthermore the two-point correlation density factorises whenever bins $t$ and $t+d$ belong to independent cascade field configurations, the averaged two-point moment finally becomes

$$
\bar{\rho}_{r, s}(d)=\frac{1}{2^{J}}\left\{\sum_{t=1}^{2^{J}-d} \rho_{r, s}(t, t+d)+\sum_{t=2^{J}-d+1}^{2^{J}} \rho_{r}(t) \rho_{s}(t+d)\right\} .
$$

Analytic expressions for $\rho_{r, s}(t, t+d)$ and $\rho_{r}(t)=\left\langle\left(\ln \varepsilon_{t}\right)^{r}\right\rangle$ are readily derived 
by inserting the cumulants (8) into the usual relations between $n$-variate moments and cumulants [6] and thence into (15).

Because they are functions of the two-point cumulants, two-point densities with $d \geq 1$ also depend on the ultrametric distance $D(t, t+d)$ between bins $t$ and $t+d$, so that (15) will contain sums of the type

$$
G_{n}(J, d)=\frac{1}{2^{J}} \sum_{t=1}^{2^{J}-d}(J-D(t, t+d))^{n} .
$$

In terms of a discrete probability distribution $p(D \mid d, J)$, proportional to the times each value of the $D$ is taken on while $t$ runs its course, this becomes

$$
G_{n}(J, d)=\sum_{D=1}^{J} p(D \mid d, J)(J-D)^{n},
$$

with $p(D \mid d, J)$ empirically found to be

$$
p(D \mid d, J)=\left\{\begin{array}{lr}
0 & (1 \leq D<A) \\
1-\left(d / 2^{A}\right) & (D=A) \\
d / 2^{D} & (A<D \leq J)
\end{array}\right.
$$

where $A=\left\lceil\log _{2} d\right\rceil$ is the ceiling of $\log _{2} d$. Insertion of (18) into (17) leads to analytical expressions for the geometrical coefficients

$$
\begin{aligned}
& G_{0}(J, d)=\left(1-2^{-J} d\right), \\
& G_{1}(J, d)=(J-A)-2 d\left(2^{-A}-2^{-J}\right), \\
& G_{2}(J, d)=(J-A)^{2}-4 d(J-A) 2^{-A}+6 d\left(2^{-A}-2^{-J}\right),
\end{aligned}
$$

which in turn yield analytical results for the averaged two-point densities $\bar{\rho}_{r, s}(d)$. From these, spatially homogeneous two-point cumulants are constructed via the inversion formulae [6]

$$
\begin{aligned}
& \bar{C}_{1,1}(d)=\bar{\rho}_{1,1}(d)-\bar{\rho}_{1}^{2}, \\
& \bar{C}_{2,1}(d)=\bar{\rho}_{2,1}(d)-2 \bar{\rho}_{1} \bar{\rho}_{1,1}(d)-\bar{\rho}_{2} \bar{\rho}_{1}+2 \bar{\rho}_{1}^{3}, \\
& \bar{C}_{3,1}(d)=\bar{\rho}_{3,1}(d)-3 \bar{\rho}_{1} \bar{\rho}_{2,1}(d)-\bar{\rho}_{3} \bar{\rho}_{1}-3 \bar{\rho}_{2} \bar{\rho}_{1,1}(d)+6 \bar{\rho}_{1}^{2} \bar{\rho}_{1,1}(d)+6 \bar{\rho}_{2} \bar{\rho}_{1}^{2}-6 \bar{\rho}_{1}^{4}, \\
& \bar{C}_{2,2}(d)=\bar{\rho}_{2,2}(d)-4 \bar{\rho}_{1} \bar{\rho}_{2,1}(d)-2\left(\bar{\rho}_{1,1}(d)\right)^{2}-\bar{\rho}_{2}^{2}+8 \bar{\rho}_{1}^{2} \bar{\rho}_{1,1}(d)+4 \bar{\rho}_{2} \bar{\rho}_{1}^{2}-6 \bar{\rho}_{1}^{4} .
\end{aligned}
$$

With Eqs. (15)-(22) we arrive for $\bar{C}_{r, 1}(d)$ at

$$
\bar{C}_{r, 1}(d)=G_{1}(J, d) c_{r+1}+G_{0}(J, d) c_{r, 1} .
$$


This turns out to be equivalent to direct translational averaging of (8), i.e. $2^{-J} \sum_{t=1}^{2^{J}} C_{r, 1}(t, t+d)$. For $s \neq 1$, however, such direct averaging is wrong and the full conversion from cumulant to moment to averaged moment and back to averaged cumulant is mandatory. For $r=s=2$ we get, for example,

$$
\begin{aligned}
\bar{C}_{2,2}(d)= & G_{1}(J, d)\left(c_{4}+4 c_{2} c_{1,1}\right)+G_{0}(J, d)\left(c_{2,2}+2 c_{1,1}^{2}\right) \\
& -2\left[G_{1}(J, d) c_{2}+G_{0}(J, d) c_{1,1}\right]^{2}+2 G_{2}(J, d) c_{2}^{2}
\end{aligned}
$$

where the additional terms are a consequence of the quadratic $\left(\bar{\rho}_{1,1}(d)\right)^{2}$ term in the expression for $\bar{C}_{2,2}(d)$ in $(22)$.

Equation (23) has a simple structure, consisting of two terms each of which is the product of a geometrical prefactor and a branching cumulant. We note that the geometrical prefactor $G_{n}(J, d)$ depends only on the geometric structure of the cascade but not on the cascade generator $p\left(q_{L}, q_{R}\right)$; in particular, as $G_{n}$ is independent of the cumulant order $r$, it is the same for all $\bar{C}_{r, 1}$. The cascade generator, on the other hand, enters only via the branching cumulants $c_{r+1}$ and $c_{r, 1}$.

For factorised cascade generators (9), eq. (23) simplifies even further to $\bar{C}_{r, 1}(d)=$ $G_{1}(J, d) c_{r+1}$. The distinguishability of $c_{r+1}$ 's for the three model generators (10)-(12) therefore implies that two-point cumulants $\bar{C}_{r, 1}(d)$ of the models will differ significantly also as they amplify $c_{r+1}$ by the geometrical prefactor.

In order to check whether our analytical results remain statistically significant for finite data samples, we simulated a chain of $m=10^{7}$ cascade configurations mimicking a time series of $m$ integral lengths, corresponding in size to a typical experimental data set. The length of the inertial cascade range was set to $L / \eta=2^{8}$. One- and two-point densities were sampled according to Eq. (14) and then converted into two-point cumulants using relations (22). Variances on translationally averaged moments were estimated for each $d$ by picking, with a random $t$, just one pair of energy fluxes $\left(\varepsilon_{t}, \varepsilon_{t+d}\right)$ per two adjacent cascade configurations, thereby avoiding correlations between picked pairs. Variances for $\bar{C}_{r, s}$ were calculated from (22) using standard error propagation.

Results for $\bar{C}_{2,1}$ are depicted in Fig. 2, with the solid lines representing the analytical function $G_{1}(J, d) c_{3}$ and simulations yielding the shaded bands. Based on Fig. 2, it would appear that statistically significant values for same-lineage cumulants $c_{r+1}$ up to at least $r=2$ can likely be extracted experimentally. As standard errors increase with increasing order, meaningful statements on higher orders become successively more difficult.

While one-point cumulants $\bar{C}_{n}$ should no doubt be measured, the two-point cumulants contain significant additional information. 
Firstly, one-point cumulants cannot distinguish between the ordered chain of cascade configurations and its spatially randomised equivalent, while two-point cumulants can do so. This is true because for the randomised case two-point cumulants are simply zero while in the ordered case they retain memory of the ordered cascade tree through the geometric coefficients $G_{n}(J, d)$, which according to relation (17) can be understood as $n$-th order moments of the probability distribution $p(D \mid d, J)$. Thus, two-point cumulants yield information on the cascade generator via the branching cumulants $c_{r+1}$ as well as testing the spatially nested cascade hierarchy via the geometric coefficients $G_{n}(J, d)$. To date, the probing of the treelike structure of the underlying process has not received much attention in the literature; we only know of Refs. [10,11], which discuss multifractal phase-transition-like behaviour for two-point densities, and of Ref. [12], which concerns itself with wavelet scale-scale correlations.

Secondly, when cascade generators $p\left(q_{L}, q_{R}\right)$ do not factorise as in (9), the splitting cumulant $c_{r, s}$ is nonzero, and so same-lineage cumulants plus multifractal scaling exponents are insufficient for their complete reconstruction. Indeed, more realistic cascade generators, attempting as best possible to reproduce the observed one-dimensional energy dissipation data, will typically exhibit small but nonzero residual correlations between $q_{L}$ and $q_{R}$. This expectation follows from a projection argument [4] stating that the energy cascade evolves in three dimensions but is observed only in one. Through their dependence on a nonzero $c_{r, s}$, the $\bar{C}_{r, s}$ should thus provide a more complete characterisation of the cascade generator.

To conclude: In the pursuit of finer statistical facets of the energy dissipation field in fully developed turbulence, translationally averaged cumulants of logarithmic field amplitudes appear to be a promising new tool. We have demonstrated for random multiplicative cascade processes that two-point cumulants can be written as simple products of geometrical coefficients times cumulant moments of the cascade generator and can thus distinguish between cascade generators which have more or less identical lowest-order multifractal scaling exponents and multiplier distributions. Through their dependence on the cascade geometry, two-point cumulants are also able to test the treelike cascade structure.

It is tempting to apply two-point cumulants of $\ln \varepsilon$ directly to the experimental energy dissipation field deduced from hot-wire time series to learn about the best approximate random multiplicative cascade model and then to study possible dependences on the Reynolds number and the flow configuration. We suggest, however, that studies of other and more elaborate models such as hierarchical shell models [11], effects of finite inertial range etc. be undertaken before this new tool is applied to data. Also, practical problems such as choosing the finest resolution scale for the analysis and finite sample size effects should be pondered in more detail. 
The authors acknowledge fruitful discussions with Jürgen Schmiegel, Jochen Cleve and Jahanshah Davoudi. This work was funded in part by the South African National Research Foundation. HCE thanks the MPIPKS for kind hospitality and support.

\section{References}

[1] A.S. Monin and A.M. Yaglom, Statistical Fluid Mechanics, Vol. 1 and 2, (MIT Press, Cambridge, 1971); U. Frisch, Turbulence (Cambridge University Press, Cambridge, 1995).

[2] C. Meneveau and K.R. Sreenivasan, J. Fluid Mech. 224, 429 (1991); K.R. Sreenivasan and R.A. Antonia, Ann. Rev. Fluid Mech. 29, 435 (1997).

[3] K.R. Sreenivasan and G. Stolovitzky, J. Stat. Phys. 78, 311 (1995); G. Pedrizzetti, E.A. Novikov and A.A. Praskovsky, Phys. Rev. E53, 475 (1996); J. Molenaar, J. Herweijer, and W. van de Water, Phys. Rev. E52, 496 (1995).

[4] B. Jouault, P. Lipa, and M. Greiner, Phys. Rev. E59, 2451 (1999); B. Jouault, M. Greiner, and P. Lipa, Physica D136, 125 (2000); B. Jouault, J. Schmiegel, and M. Greiner, chao-dyn/9909033.

[5] M. Greiner, H.C. Eggers, and P. Lipa, Phys. Rev. Lett. 80, 5333 (1998); M. Greiner, J. Schmiegel, F. Eickemeyer, P. Lipa, and H.C. Eggers, Phys. Rev. E58, 554 (1998).

[6] P. Carruthers, H.C. Eggers, and I. Sarcevic, Phys. Lett. B254, 258 (1991).

[7] M. Greiner, J. Giesemann, and P. Lipa, Phys. Rev. E56, 4263 (1997).

[8] A. Naert, R. Friedrich, and J. Peinke, Phys. Rev. E56, 6719 (1997); P. Marcq and A. Naert, Physica D134, 368 (1998).

[9] J. Cleve and M. Greiner, Phys. Lett. A273, 104 (2000).

[10] C. Meneveau and A. Chhabra, Physica A164, 564 (1990); J. O'Neil and C. Meneveau, Phys. Fluids A5, 158 (1993).

[11] R. Benzi, L. Biferale, R. Tripiccione, and E. Trovatore, Phys. Fluids 9, 2355 (1997); R. Benzi, L. Biferale, and E. Trovatore, Phys. Rev. Lett. 79, 1670 (1997).

[12] A. Arneodo, E. Bacry, S. Manneville, and J.F. Muzy, Phys. Rev. Lett. 80, 708 (1998). 

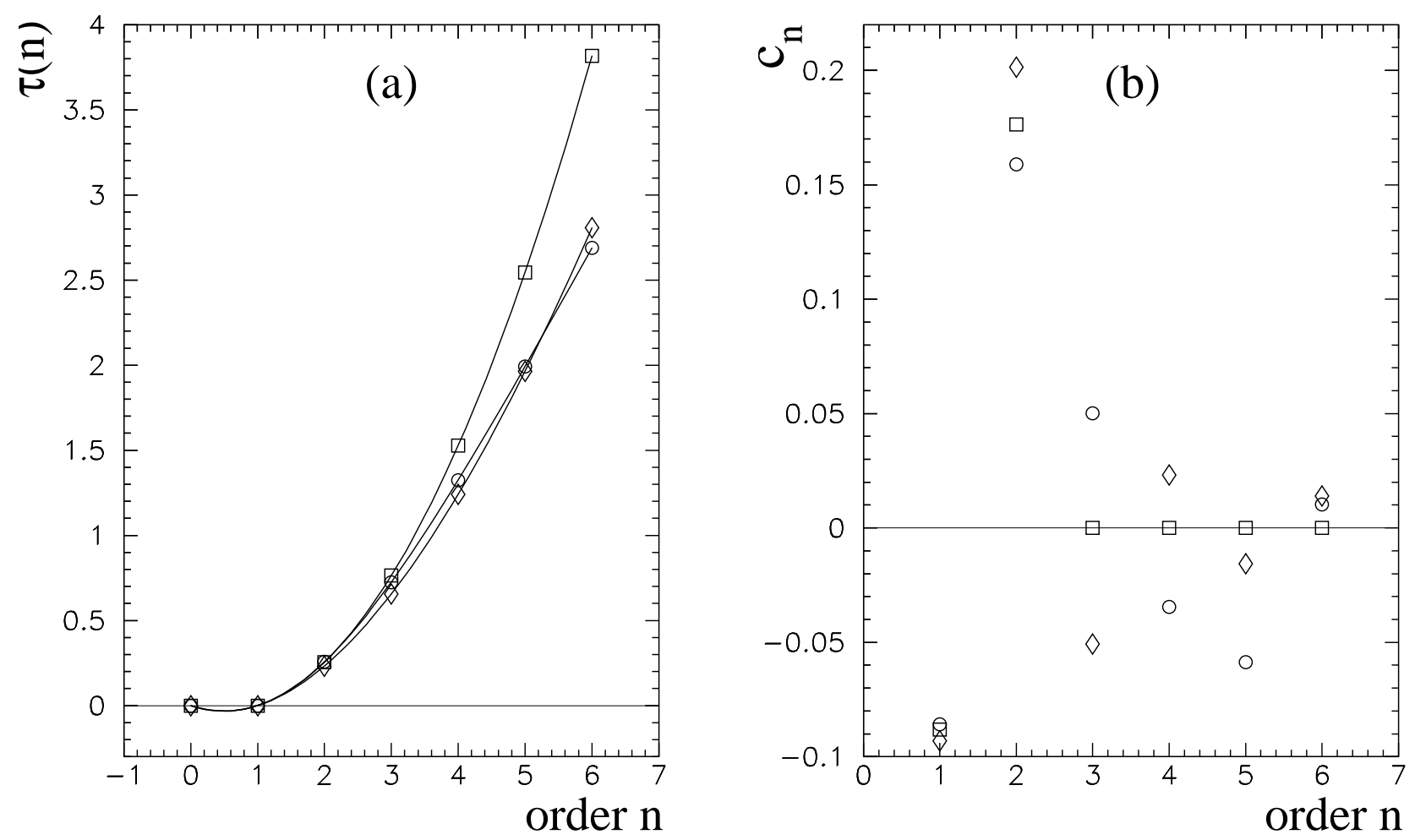

Figure 1: (a) Multifractal scaling exponents and (b) same-lineage cumulants for the binomial (circles), lognormal (squares), and beta (diamonds) cascade generators (10)-(12). 


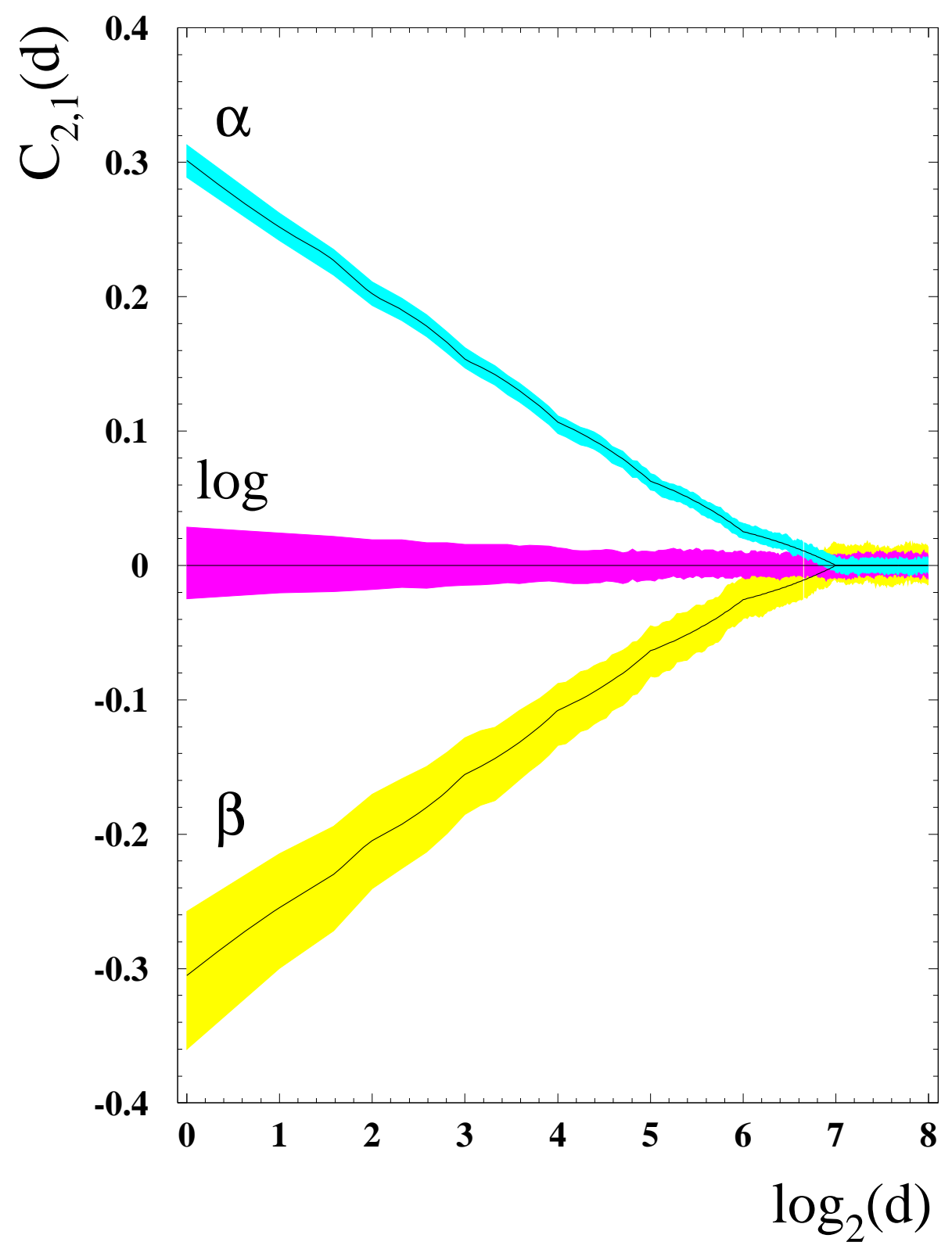

Figure 2: Analytical (solid lines) and simulated (shaded bands) two-point cumulant $\bar{C}_{2,1}(d)$ as a function of bin-bin distance $d$, for the binomial (top), lognormal (middle) and beta (bottom) cascade generators (10)-(12). 